\title{
Predictors of diagnostic yield in bronchoscopy: a retrospective cohort study comparing different combinations of sampling techniques
}

\author{
Kjetil Roth*1,2, Jon A Hardie ${ }^{1}$, Alf H Andreassen ${ }^{1}$, Friedemann Leh ${ }^{3}$ and \\ Tomas ML Eagan ${ }^{1}$
}

Address: ${ }^{1}$ Dept. of Thoracic Medicine, Haukeland University Hospital, Bergen, Norway, ${ }^{2}$ Dept. of Internal Medicine, Aalesund Hospital, Aalesund, Norway and ${ }^{3}$ Dept. of Pathology, Haukeland University Hospital, Bergen, Norway

Email: Kjetil Roth* - kjetil.roth@med.uib.no; Jon A Hardie - Jon.Hardie@med.uib.no; Alf H Andreassen - Alf.Andreassen@ @else-bergen.no; Friedemann Leh - Friedemann.Leh@helse-bergen.no; Tomas ML Eagan - Tomas.Eagan@med.uib.no

* Corresponding author

Published: 26 January 2008

BMC Pulmonary Medicine 2008, 8:2 doi:10.1 I86/147/-2466-8-2

This article is available from: http://www.biomedcentral.com/I47I-2466/8/2

(C) 2008 Roth et al; licensee BioMed Central Ltd.

This is an Open Access article distributed under the terms of the Creative Commons Attribution License (http://creativecommons.org/licenses/by/2.0), which permits unrestricted use, distribution, and reproduction in any medium, provided the original work is properly cited.

\begin{abstract}
Background: The reported diagnostic yield from bronchoscopies in patients with lung cancer varies greatly. The optimal combination of sampling techniques has not been finally established.

The objectives of this study were to find the predictors of diagnostic yield in bronchoscopy and to evaluate different combinations of sampling techniques.

Methods: All bronchoscopies performed on suspicion of lung malignancy in 2003 and 2004 were reviewed, and 363 patients with proven malignant lung disease were included in the study. Sampling techniques performed were biopsy, transbronchial needle aspiration (TBNA), brushing, small volume lavage (SVL), and aspiration of fluid from the entire procedure. Logistic regression analyses were adjusted for sex, age, endobronchial visibility, localization (lobe), distance from carina, and tumor size.
\end{abstract}

Results: The adjusted odds ratios (OR) with $95 \%$ confidence intervals (Cl) for a positive diagnostic yield through all procedures were 17.0 (8.5-34.0) for endobronchial lesions, and 2.6 (I.3-5.2) for constriction/compression, compared to non-visible lesions; 3.8 (1.3-10.7) for lesions $>4 \mathrm{~cm}, 6.7$ (2.I-2I.8) for lesions $3-4 \mathrm{~cm}$, and $2.5(0.8-7.9)$ for lesions $2-3 \mathrm{~cm}$ compared with lesions $<=2 \mathrm{~cm}$. The combined diagnostic yield of biopsy and TBNA was $83.7 \%$ for endobronchial lesions and $54.2 \%$ for the combined group without visible lesions. This was superior to either technique alone, whereas additional brushing, SVL, and aspiration did not significantly increase the diagnostic yield.

Conclusion: In patients with malignant lung disease, visible lesions and larger tumor size were significant predictors of higher diagnostic yield, after adjustment for sex, age, distance from carina, side and lobe. The combined diagnostic yield of biopsy and TBNA was significant higher than with either technique alone. 


\section{Background}

The incidence of lung cancer in Norway increased from $21.1 / 100000$ person years in $1967-1971$ to $36.1 / 100000$ in 2000-2004 for men and from 4.5/100 000 to $21.1 / 100$ 000 for women [1]. Bronchoscopy is the main diagnostic procedure in patients with endoscopic visible lesions. British Thoracic Society (BTS) guidelines from 2001 recommend biopsies, brushings, and washings for sampling from visible lesions. The diagnostic yield for visible lesions should be at least $80 \%$ [2]. Transbronchial needle aspiration (TBNA) was not included in the recommendation, and the optimal combination of sampling techniques in peripheral lesions was not settled [2].

Computer tomography (CT) guided sampling techniques have a diagnostic yield of approximately $90 \%$ in peripheral lung cancer [3], with the disadvantage of a high incidence of pneumothorax [4]. Previous studies of bronchoscopy in peripheral lesions have shown a great variability in the diagnostic yield, with sensitivity for cancer between $20 \%$ and $86 \%$ [5-9]. The reported predictors of positive samples have been size [6,9-16], location $[10,13,14,17]$, visible lesion, compression or constriction $[12,18,19]$, CT bronchus sign $[13,17,20]$, fuzzy or sharp border [11], the use of a C-arm fluoroscope [8,21], and sampling technique $[11,14,15,18,22-29]$. Many studies were based on bronchoscopies performed by selected investigators in highly specialized centres $[5,8,10,18]$.

The great variability in the previous studies makes it difficult to know if the real life situation in a clinical practice is comparable with the reported results. The choice of sampling techniques is often left to the physician who performs the bronchoscopy, and it is not known if a standardised approach gives better results.

The aims of this study were to evaluate the sensitivity of bronchoscopy for detecting malignant disease in clinical practice, identify predictors of a high diagnostic yield, and to evaluate different pairs of sampling techniques.

\section{Methods}

All 1438 bronchoscopies performed between January 2003 and December 2004 at Haukeland University Hospital, Bergen, Norway, were retrospectively reviewed. All procedures where the indication for bronchoscopy was to obtain samples from a lesion suspicious of malignancy and where the final diagnosis obtained through all possible methods was malignant lung disease were eligible for inclusion in the study. If a patient had repeated bronchoscopies, only the first bronchoscopy was included.

Of 493 patients with a lesion suspicious of malignancy, 367 patients were investigated with bronchoscopy of later proven malignant disease. Three patients were excluded because no samplings had been performed during bronchoscopy, and one patient was excluded because it was not possible to perform the bronchoscopy. Thus, 363 patients were included in the study sample.

Twenty three medical doctors; nine pulmonologists and fourteen trainees (pulmonary residents and fellows) performed the bronchoscopies. The investigations were performed with Olympus BF 1T 160 bronchoscopes, using Boston "Radial Jaw3" for biopsies, Boston 21 Gauche "Stifcor" transbronchial aspiration needle for TBNA, and Boston "Cellebrity" for brushings. TBNA was taken directly from endobronchial lesions, under visual control from constriction and compression, and blind or under fluoroscopic guidance from peripheral lesions. The procedures were performed transorally without an endotracheal tube. Patients were semi-sedated with pethidine hydrochloride 25-75 mg or midazolam 2.5-5 mg. Biopsies and small volume lavage (SVL), a bronchial washing with 10-20 ml saline were fixated in formalin. TBNA and brushings were alcohol fixed on a glass slide. In addition, aspiration from the whole bronchoscopy was collected and a sample of 10-20 ml was fixated in formalin.

Two of the authors (KR and TME) registered endobronchial visibility and indications for bronchoscopy based on a review of the bronchoscopy reports. Endobronchial visibility was categorized into 1) visible lesion, 2) constriction, compression or suspected submucosal changes, or 3) non-visible lesion. The largest size of the lesion was measured from the CT scan in all but five cases, in which size was estimated from the chest radiograph. The distance from carina to the lesion was measured on a posterior-anterior chest radiograph or on a reconstruction from the CT scan. In 40 cases the distance from carina was impossible to measure. The localization (side and lobe) of the lesion was registered from the CT scan. In cases of multiple lesions, the sampled lesions were registered. The cases were categorized as indeterminate when it was impossible to decide which lesion that had been sampled.

Malignant lung disease was defined as positive histological or cytological results or certain malignant disease after clinical follow up. The Department of Pathology provided a computer-based search in the registry of systemized nomenclature of medicine (SNOMED) codes from all bronchoscopies, ultrasound guided transthoracic needle aspirations, CT guided samplings, and operations. Cells suspicious of malignancy usually lead to further investigations and were not included in the definition of positive diagnostic yield. The diagnostic yield in this study was defined as sensitivity for cancer. The gold standard was defined as histological proven malignant disease or clinical malignant disease during follow up. Three sources of information were used to avoid exclusion of patients with 
later proven malignant disease: 1) A computer based search through patient journals for a later malignant diagnosis. 2) A review of the journals of all patients who died before November 2005. 3) Follow up until November 2005 of all patients discharged with a diagnosis of an uncertain pulmonary lesion.

The statistical analyses were performed in SPSS, using Chi square tests for univariate analyses, multivariate logistic regression to estimate the odds ratios and adjust for confounding, and McNemar's test to compare different sampling techniques. The Regional Norwegian Ethical Committee and the Norwegian Social Science Data Service approved the study.

\section{Results}

The baseline characteristics of the patients are displayed in Table 1. The first bronchoscopy provided a conclusive diagnosis of malignant disease in 161 of the 363 patients $(44 \%)$. Two patients had cytological specimens suspi-

Table I: Baseline characteristics of 363 cases

\begin{tabular}{|c|c|c|}
\hline & $\mathrm{n}$ & $\%$ \\
\hline \multicolumn{3}{|l|}{ Sex } \\
\hline Male & 221 & 60.9 \\
\hline Female & 142 & 39.1 \\
\hline \multicolumn{3}{|l|}{ Age (years) } \\
\hline$<59$ & 87 & 24.0 \\
\hline $59-67$ & 92 & 25.3 \\
\hline $68-74$ & 90 & 24.8 \\
\hline$>74$ & 94 & 25.9 \\
\hline \multicolumn{3}{|l|}{ Size* } \\
\hline$<=2 \mathrm{~cm}$ & 41 & 11.3 \\
\hline $2-3 \mathrm{~cm}$ & 62 & 17.1 \\
\hline $3-4 \mathrm{~cm}$ & 53 & 14.6 \\
\hline$>4 \mathrm{~cm}$ & 207 & 57.0 \\
\hline \multicolumn{3}{|l|}{ Distance from carina\# } \\
\hline$<=5 \mathrm{~cm}$ & 144 & 39.7 \\
\hline$>5 \mathrm{~cm}$ & 179 & 49.3 \\
\hline Indeterminate & 40 & 11.0 \\
\hline \multicolumn{3}{|l|}{ Endobronchial visibility } \\
\hline Non visible lesion & 132 & 36.4 \\
\hline Constriction, compression or submucosal lesion & 90 & 24.8 \\
\hline Visible lesion & $|4|$ & 38.8 \\
\hline \multicolumn{3}{|l|}{ Localization } \\
\hline Left upper lobe without lingula & 75 & 20.7 \\
\hline Left lingula & 10 & 2.8 \\
\hline Left lower lobe & 53 & 14.6 \\
\hline Right upper lobe & 88 & 24.2 \\
\hline Right middle lobe & 19 & 5.2 \\
\hline Right lower lobe & 73 & 20.1 \\
\hline Mediastinum & 30 & 8.3 \\
\hline Indeterminate & 15 & 4.1 \\
\hline
\end{tabular}

*Size was measured on axial $\mathrm{Ct}$ thorax, only chest radiograph available in 5 cases.

\#Distance from carina is measured as the distance from carina to the lesion on the chest radiograph, front projection. cious of cancer in the first bronchoscopy, with no other sampling techniques to confirm the diagnosis. Almost $40 \%$ of the patients diagnosed with cancer these two years were women.

The final diagnostic method and pathological classification is shown in Table 2. Transthoracic sampling techniques provided the diagnosis in $105 / 363$ patients. Of the transthoracic samples, 87 were obtained by CT guided sampling, 12 by ultrasound guided sampling, two were pleural biopsies, and four were pleural effusions (Table 2).

Table 3 presents the diagnostic yield of the different bronchoscopic sampling techniques. The sampling techniques performed were biopsy (201/363), TBNA (191/363), brushing (187/363), SVL (72/363), and aspiration of fluid from the entire procedure (356/363). Biopsy consistently gave the highest diagnostic yield with the possible exception in the case of lesions smaller than $2 \mathrm{~cm}$. In univariate analyses endobronchial visibility, tumor size, and distance from carina were predictors of a higher diagnostic yield (Table 3). The overall sensitivity for cancer increased from $16.7 \%$ in non-visible lesions, to $34.4 \%$ for compression, constriction or submucosal disease, and further to $76.6 \%$ in visible lesions $\left(\chi^{2}: \mathrm{p}<0.001\right)$.

In non visible lesions the diagnostic yield using a C-arm fluoroscope was $17 / 48$ (35.4\%) compared to $4 / 83(4.8 \%)$

Table 2: The diagnostic method and final diagnosis of 363 cases with malignant lung disease.

\begin{tabular}{lcc}
\hline & $\mathrm{n}$ & $\%$ \\
\hline Final diagnostic method & & \\
First bronchoscopy* & 163 & 44.9 \\
Repeated bronchoscopy & 28 & 7.7 \\
Transthoracic sampling & 105 & 28.9 \\
Mediastinoscopy & 2 & 0.6 \\
Operation, autopsy and open lung biopsy & 11 & 3.0 \\
Sampling from other organs than the lung & 15 & 4.1 \\
Clinical diagnosis of cancer & 17 & 4.7 \\
Malignant diagnosis obtained before bronchoscopy & 22 & 6.1 \\
Pathology & & \\
Small cell lung cancer & 53 & 14.6 \\
Adenocarcinoma & 100 & 27.5 \\
Squamous cell carcinoma & 66 & 18.2 \\
Large cell carcinoma & 10 & 2.8 \\
Non classifiable non small cell lung cancer. & 82 & 22.6 \\
Metastasis to the lung & 21 & 5.8 \\
Other cancer in the lung\# & 14 & 3.9 \\
Only clinical diagnosis & 17 & 4.7 \\
\hline
\end{tabular}

* 2 patients diagnosed with uncertain pathology.

\#Other cancer in the lung: Carcinoid tumor:6, Lymphoma:5, Mesothelioma:2,

Neuroendocrine tumor:I. 
Table 3: Diagnostic yield of different sampling techniques

\begin{tabular}{|c|c|c|c|c|c|c|}
\hline & Biopsy $(n=20 \mathrm{I})$ & TBNA $(n=191)$ & Brushing $(n=187)$ & $S V L(n=72)$ & Aspiration $(n=356)$ & All $(n=363)$ \\
\hline Overall diagnostic yield & $|22 / 20|(60.7)$ & $78 / 191$ (40.8) & $43 / 187(23.0)$ & $5 / 72(6.9)$ & $29 / 356(8.1)$ & $161 / 363(44.4)$ \\
\hline Endobronchial visibility & ** & $* *$ & ** & & ** & $* *$ \\
\hline Non visible lesion & $9 / 36(25.0)$ & $4 / 21(19.0)$ & $13 / 68(16.0)$ & $3 / 46(6.5)$ & $5 / 130(3.8)$ & $22 / 132(16.7)$ \\
\hline Constriction/compression & $22 / 48(45.8)$ & $17 / 69(24.6)$ & $5 / 49(10.2)$ & I/II (9.I) & $2 / 88(2.3)$ & $31 / 90(34.4)$ \\
\hline Visible lesion & $91 / 117(77.8)$ & $57 / 101(56.4)$ & $25 / 57(43.9)$ & $\mathrm{I} / \mathrm{I} 5(6.7)$ & $22 / 138(15.9)$ & $108 / 141(76.6)$ \\
\hline Tumor size & $*$ & & & & & $* *$ \\
\hline$<=2 \mathrm{~cm}$ & $3 / 13(23.1)$ & $4 / 16(25.0)$ & $2 / 19(10.5)$ & $0 / 13(0.0)$ & $2 / 4 \mid(4.9)$ & $7 / 4 \mid(\mid 7.1)$ \\
\hline$>2 \mathrm{~cm}$ and $<=3 \mathrm{~cm}$ & $15 / 24(62.5)$ & $6 / 18(25.0)$ & 4/35 (II.4) & $0 / 19(0.0)$ & $6 / 59(10.2)$ & $20 / 62(32.3)$ \\
\hline$>3 \mathrm{~cm}$ and $<=4 \mathrm{~cm}$ & $19 / 35(54.3)$ & $12 / 24(50.0)$ & $8 / 31$ (25.8) & I/I3 (7.7) & $4 / 5 I(7.8)$ & $25 / 53(47.2)$ \\
\hline$>4 \mathrm{~cm}$ & $85 / 129(65.9)$ & $56 / 127(44.1)$ & $29 / 102(28.4)$ & $4 / 27(14.8)$ & $17 / 205(8.3)$ & $109 / 207(52.7)$ \\
\hline Distance to carina & $*$ & & $*$ & & & $*$ \\
\hline$<=5 \mathrm{~cm}$ & $59 / 86(68.6)$ & $40 / 93(43.0)$ & $23 / 68(33.8)$ & $0 / 12(0.0)$ & $13 / 142(9.2)$ & $78 / \mid 44(54.2)$ \\
\hline$>5 \mathrm{~cm}$ & $44 / 88(50.0)$ & $26 / 74(35.1)$ & $17 / 102(16.7)$ & $3 / 52(5.8)$ & $12 / 165(6.8)$ & $62 / 179(34.6)$ \\
\hline Indeterminate & 19/27 (70.4) & $12 / 24(50.0)$ & $3 / 17(17.6)$ & $2 / 8(25.0)$ & $4 / 37(10.8)$ & $21 / 40(52.5)$ \\
\hline \multicolumn{7}{|l|}{ Side } \\
\hline Left & $49 / 80$ (6I.3) & $26 / 75(34.7)$ & $20 / 80(25.0)$ & $4 / 27(14.8)$ & $14 / 147(9.5)$ & $62 / I 5 I(4||)$. \\
\hline Right & $68 / 115(59.1)$ & $46 / 106(43.4)$ & $22 / 103(21.4)$ & $\mathrm{I} / 42(2.4)$ & $14 / 195(7.2)$ & $92 / 198(46.5)$ \\
\hline Indeterminate & $5 / 6(83.3)$ & $6 / 10(60.0)$ & I/4 (25.0) & $0 / 3(0.0)$ & $1 / 14(7.1)$ & $7 / 14(50.0)$ \\
\hline \multicolumn{7}{|l|}{ Lobe } \\
\hline Upper lobe without lingula & $47 / 92(51.1)$ & 29/9I (31.9) & $20 / 92(21.7)$ & 3/27 (II.I) & $15 / 162(9.3)$ & $68 / 164(4 I .5)$ \\
\hline Middle lobe/lingula & $8 / 13(61.5)$ & $6 / 14(42.9)$ & $6 / 18(33.3)$ & $0 / 9(0.0)$ & $0 / 27(0.0)$ & I I/29 (37.9) \\
\hline Lower lobe & $50 / 74(67.6)$ & $29 / 58(50.0)$ & $13 / 60(21.7)$ & $2 / 30(6.7)$ & $1 \mathrm{I} / \mathrm{I} 24(8.9)$ & $61 / 127(48.0)$ \\
\hline Mediastinum & $8 / 11(72.7)$ & $7 / 18(38.9)$ & $2 / 13(15.4)$ & $0 / 5(0.0)$ & $0 / 30(0.0)$ & $12 / 30(40.0)$ \\
\hline Indeterminate & $9 / 11(81.8)$ & $7 / 10(70)$ & $2 / 4(50.0)$ & $0 / /(0.0)$ & $3 / 13(23.1)$ & $9 / 13(69.2)$ \\
\hline
\end{tabular}

$*_{p}<0.05 . *^{*} p<0.001$

Data are presented as number of positive samples/all samples (\%). Statistical analysis: $\chi^{2}$.

TBNA = Transbronchial needle aspiration. SVL = Small volume lavage.

without a C-arm fluoroscope $\left(\chi^{2}: \mathrm{p}<0.001\right)$, data not shown in Table 3.

Table 4 presents predictors of diagnostic yield for each sampling technique in a multivariate analysis after adjustment for age, sex, lobe, endobronchial visibility, size and distance from carina. The adjusted odds ratio (OR) for a positive diagnostic result, was $2.6(1.3-5.2)$ for constriction, compression or suspected submucosal changes and 17.0 (8.5-34.0) for visible lesions, compared to non visible lesions. Endobronchial visibility was a significant predictor for a higher diagnostic yield in all sampling techniques. Larger tumor size predicted an overall higher diagnostic yield, but was statistically significant only for

Table 4: Predictors of a higher diagnostic yield. Odds ratio $(95 \% \mathrm{Cl})$ in multivariate analysis.

\begin{tabular}{|c|c|c|c|c|c|}
\hline & Biopsy $(n=201)$ & TBNA $(n=191)$ & Brushing $(n=187)$ & Aspiration $(n=356)$ & All $(n=363)$ \\
\hline Endobronchial visibility & $* *$ & $* *$ & $*$ & $* *$ & $* *$ \\
\hline Non-visible lesion & I & I & I & I & I \\
\hline Compression/constriction & $2.4(0.8-7.0)$ & $1.2(0.3-4.4)$ & $0.4(0.1-\mid .5)$ & $0.8(0.1-4.6)$ & $2.6(1.3-5.2)$ \\
\hline Visible lesion & $10.8(3.8-30.7)$ & $5.0(1.4-17.5)$ & $3.1(1.1-8.3)$ & 6.4 (1.9-20.9) & $17.0(8.5-34.0)$ \\
\hline Tumor size & $*$ & & & & $*$ \\
\hline$<=2 \mathrm{~cm}$ & I & I & I & I & I \\
\hline $2-3 \mathrm{~cm}$ & $7.9(1.4-45.1)$ & $0.8(0.2-4.0)$ & I.I (0.2-7.3) & $2.2(0.4-12.7)$ & $2.5(0.8-7.9)$ \\
\hline $3-4 \mathrm{~cm}$ & $9.4(1.7-52.2)$ & $3.1(0.6-15.3)$ & $2.8(0.5-17.0)$ & $2.1(0.3-13.6)$ & $6.7(2.1-21.8)$ \\
\hline$>4 \mathrm{~cm}$ & $6.7(1.5-30.6)$ & $2.0(0.5-7.6)$ & $2.0(0.4-10.9)$ & $1.0(0.2-5.4)$ & $3.8(1.3-10.7)$ \\
\hline
\end{tabular}

Odds ratio adjusted for age, sex, lobe and distance from carina. TBNA = Transbronchial needle aspiration.

* Likelihood ratio: $p<0.05$. ** Likelihood ratio: $p<0.001$. 
biopsies. In the multivariate analyses, distance from carina and localization of the lesion did not predict the diagnostic yield.

Different pairs of sampling techniques were compared (Table 5). The sample size was not large enough to compare endobronchial visibility in three categories. Therefore non-visible lesions, compression, constriction, or suspected submucosal changes were combined to one category. In 86 patients with visible lesions, biopsy and TBNA were performed with a combined diagnostic yield of $83.7 \%(72 / 86$, Table 5$)$. In 38 of the 86 procedures, brushing was performed in addition to biopsy and TBNA, and in 85 of the procedures aspirations were also examined. Cytological examination of the brushings and aspirations provided an increased diagnostic yield of one case (NS). Compared to the combination of biopsy and TBNA in these 86 procedures, seven cases would have been missed without TBNA ( $p=0.02$ ), and 22 cases would have been missed without biopsy $(\mathrm{p}<0.001)$. The result in the group with non-visible lesions, compression, constriction or suspected submucosal changes was similar. In this group, biopsy and TBNA was performed in 48 patients with a combined diagnostic yield of $54.2 \%$ (Table 5). In these procedures additional 47 aspirations and 25 brushings were performed, which increased the diagnostic yield by only one case (NS). The diagnostic yield with a combination of biopsy and TBNA was significantly higher than with biopsy or TBNA alone.

\section{Discussion}

Endobronchial visibility and tumor size were the predictors of diagnostic yield in bronchoscopies of patients with malignant disease in this study. The diagnostic yield was $16.7 \%$ in procedures with non-visible lesions, $34.4 \%$ in procedures with compression, constriction or suspected submucosal changes, and $76.6 \%$ in procedures with endobronchial visible lesions. The combination of biopsy and TBNA had the highest diagnostic yield both in visible lesions and in the combined group of non-visible lesions, constriction, compression, or suspected submucosal changes. Biopsy and TBNA together was significant better than either technique alone.

There are some methodological issues to consider.

The main strength of this study is that it reflects the diagnostic value of bronchoscopy in a regular clinical practice. For many operative procedures, an important factor is the skill of the operator. The current study did not have the power to examine diagnostic yield by operator. However, the large number of operators partly reflects the large number of patients seen with lung cancer, at our centre. The results of this study should be comparable to centres that include trainees and where each doctor performs approximately thirty bronchoscopies per year.

Patient selection bias is a problem in studies of diagnostic yield in bronchoscopy. Haukeland University Hospital is the only centre for diagnosing lung cancer in Hordaland County and some smaller surrounding municipalities. Thus all patients from surrounding area would be included. The follow up time to December 2005 with inclusion of clinical cancer were important factors to ensure inclusion of all cancers present among those examined in 2003/04.

Another selection bias pertains to the choice of sampling methods. In our centre, the choice of sampling method is left to the judgement of the examiner. Thus, although in most bronchoscopies more than one sampling method was employed, for example biopsy and TBNA, rarely all five available techniques were used. In many procedures there is an urge to use the least amount of material and

Table 5: Diagnostic yield of different combinations of sampling techniques

\begin{tabular}{|c|c|c|c|c|c|c|c|}
\hline & \multirow{2}{*}{$\begin{array}{l}\text { The result of the first two } \\
\text { sampling techniques in the } \\
\text { combination } \\
\text { DY (\%) }\end{array}$} & \multicolumn{2}{|c|}{$\begin{array}{l}\text { The result of all sampling } \\
\text { techniques performed }\end{array}$} & \multicolumn{2}{|c|}{$\begin{array}{l}\text { The result of the first } \\
\text { sampling technique (I) }\end{array}$} & \multicolumn{2}{|c|}{$\begin{array}{l}\text { The result of the second } \\
\text { sampling technique (2) }\end{array}$} \\
\hline & & DY (\%) & $\mathrm{P}$ & DY (\%) & $P$ & DY (\%) & $\mathrm{P}$ \\
\hline \multicolumn{8}{|c|}{ Non-visible lesion/compression or constriction: } \\
\hline $\operatorname{Biopsy}(\mathrm{I})$ and TBNA(2) & $26 / 48(54.2)$ & $27 / 48(56.3)$ & NS & $20 / 48(41.7)$ & 0.03 & 16/48 (33.3) & 0.002 \\
\hline Biopsy(I) and Brushing(2) & $18 / 42(42.9)$ & $20 / 42(47.6)$ & NS & $14 / 42(33.3)$ & NS & $9 / 42(21.4)$ & 0.004 \\
\hline TBNA $(I)$ and Brushing(2) & $|6 / 5|(31.4)$ & $2|/ 5|(4 \mid .2)$ & 0.06 & ||$/ 5 \mid(2 \mid .6)$ & 0.06 & $10 / 51(19.6)$ & 0.03 \\
\hline \multicolumn{8}{|c|}{ Endobronchial visible lesion: } \\
\hline $\operatorname{Biopsy}(\mathrm{I})$ and TBNA(2) & $72 / 86(83.7)$ & $73 / 86(84.9)$ & NS & $65 / 86(75.6)$ & 0.02 & $50 / 86(58.1)$ & $<0.001$ \\
\hline Biopsy(I) and Brushing(2) & $37 / 46(80.4)$ & $39 / 46(84.8)$ & NS & $34 / 46(73.9)$ & NS & $23 / 46(50.0)$ & $<0.001$ \\
\hline TBNA(I) and Brushing(2) & $30 / 47(63.8)$ & $39 / 47(83.0)$ & 0.004 & $27 / 47(57.4)$ & NS & $22 / 47(46.8)$ & 0.008 \\
\hline
\end{tabular}

Data are presented as number of positive samples/all samples (\%). Statistical analysis: McNemar's test. DY = Diagnostic Yield. All results are compared with the diagnostic yield of the respective combination of the two sampling techniques. 
time necessary, in order to avoid complications and discomfort for the patients. Thus, an operator who feels to have obtained an adequate sample may terminate the procedure without all techniques being employed. On the other hand, some sampling techniques may be dropped if complications arise, or the tumor seems impossible to reach. Clearly this is a weakness in the current study, when comparing the different sampling methods. However, the current study reflects how bronchoscopies are normally performed. In studies where all possible techniques are used, there may be a selection bias in that patients where the procedure is terminated prior to full sampling, for instance due to complications, are excluded from the study. This bias would likely inflate the results from studies in which only patients having undergone all procedures are included.

Previous studies have categorized "compression, constriction or suspected submucosal changes" either with visible lesions or with non-visible lesions. Some studies have included these as non specific findings $[12,30]$, thus increasing the diagnostic yield of peripheral lesions. Other studies have classified the findings as submucosalperibronchial disease [31,32], decreasing the diagnostic yield of visible lesions. We have classified this group as a separate category. The finding that these lesions show a diagnostic yield intermediate between visible and nonvisible supports our choice of classification. However, for the analyses in table 5, sample size dictated that non-visible lesions be grouped with "compression, constriction or suspected submucosal changes". As TBNA and biopsy were more often used for the later category, and brushings more often for non-visible lesions, there could be a tendency for the results of TBNA to appear better in the combined category.

The overall diagnostic yield of $77 \%$ in visible lesions, is similar to previously reported results $[7,21,25,30,31]$. The overall diagnostic yield of $17 \%$ in non visible lesions is lower than in some studies [3,5-15], but higher than the yield reported in a Scottish multi-centre study (9\%) [30].

The individual diagnostic yield of biopsy, brushing or TBNA from visible lesions were similar to previous reported studies $[18,21,28,29]$. SVL and aspiration had lower diagnostic yields for visible and non visible lesions, compared with other studies $[21,25,33]$. Studies with bronchoalveolar lavage have had a higher diagnostic yield, possibly due to higher fluid volume $[22,23]$. The low diagnostic yield might also be explained by the procedure of taking a small sample of $10-20 \mathrm{ml}$ from the fluid aspirated and the lack of wedging the bronchoscope into the affected bronchus. Although there are relatively few prospective trials, they tend to report a higher diagnostic yield than retrospective trials, which may be due to the benefits of planning $[20,28]$. Studies from highly specialized centres or from selected procedures with all sampling techniques, have a higher diagnostic yield compared to the current study $[5,8,13]$.

The C-arm fluoroscope increased the diagnostic yield from $5 \%$ to $35 \%$. The rate of fluoroscopy in the current study was low, mainly because the C-arm fluoroscope was operated by radiographers who were not always available. The current study was not powered to examine differences in diagnostic yield between procedures with and without the C-arm fluoroscope. However, this study suggests a significant benefit from using the fluoroscope.

Several studies have examined predictors for a higher diagnostic yield, but only in univariate analysis [9-11,13$16,18,20]$. Significant predictors found have been size $[10,14-16,20]$, location [9-11], and endobronchial visibility [18]. In the current study, endobronchial visibility and tumor size prevailed as significant predictors when several potential predictors were examined together. Stratified on each sampling technique, the effect of size was significant only for biopsy. Both brushing and TBNA gave better results in larger lesions, but the results were not statistically significant. This could indicate that sample size was too small to show the effect of size on these sampling methods. However, brushings might be sampled from a wider area than biopsy, and the size of the lesion might be less important for this method.

Knowing the diagnostic yield of each sampling technique is important for choosing the optimal combination [11,32-34]. In the current study biopsy was the most important sampling technique in patients with and without visible lesions. Addition of TBNA or brushing increased the diagnostic yield of biopsy, but three sampling techniques were not significantly better than two. Biopsy and TBNA had the highest combined diagnostic yield but the results must be interpreted cautiously. The most important reasons that the result could be biased was the retrospective nature of the study, because non visible lesions had to be categorized together with compression and constriction, and because of the low number of biopsy, brushing and TBNA. Although brushings did not prove significant additional benefit, too few procedures included brushings for the matter to be settled. However, the value of SVL and aspiration in addition to the other techniques seemed too small to warrant the effort. While BTS guidelines presently recommend a combination of biopsy, brushing, and washing, these data suggest that the combination of biopsy and TBNA may be superior for visible and non-visible lesions. 


\section{Conclusion}

This study evaluated predictors of diagnostic yield of bronchoscopy reflecting clinical real life. Endobronchial visible lesion and larger tumor size predicted a higher diagnostic yield. The diagnostic yield was comparable with previous studies for visible lesions but was lower than in many of the previous studies for non-visible lesions.

BTS guideline recommended biopsy, brushing and washing for visible lesions. This study has shown that biopsy and TBNA might be better. In the combined group of compression, constriction, suspected submucosal disease and non visible lesions, biopsy and TBNA was better than each sampling technique alone, however this study did not have sufficient power to determine whether brushing should be performed or not. These groups should be further investigated to find the optimal combination of sampling techniques and a cost effectiveness analysis could be performed. Washings were performed in almost all procedures and did not increase the diagnostic yield significantly in any groups.

\section{Abbreviations}

BTS = British Thoracic Society, CI = confidence interval, $\mathrm{CT}=$ computer tomography, $\mathrm{OR}=$ odds ratio, $\mathrm{SD}=$ standard deviation, SNOMED = systemized nomenclature of medicine, $\mathrm{SVL}=$ small volume lavage, $\mathrm{TBNA}=$ transbronchial needle aspiration.

\section{Competing interests}

The author(s) declare that they have no competing interests.

\section{Authors' contributions}

TME and KR read the bronchoscopy reports and registered the information provided, viewed the chest radiographs and the CT scans, implemented the information from the pathological department, performed the statistical analysis, and wrote the manuscript.

JH and AHA participated in the design of the study, and reviewed the manuscript thoroughly.

FL provided all the information from the pathological department, was part of the planning of the study, and reviewed the manuscript thoroughly.

\section{Acknowledgements}

The study was founded by a one year grant for KR from Helse Vest and by a grant from Helse Sunnmore.

\section{References}

I. Cancer registry of Norway [http://www.kreftregisteret.no]. (Nov 2006).

2. Honeybourne D, Babb J, Bowie P, Brewin A, Fraise A, Garrard C, Harvey J, Lewis R, Neumann C, Wathen CG, Williams T: British
Thoracic Society guidelines on diagnostic flexible bronchoscopy. Thorax 200I, 56:II-12I.

3. Schreiber G, McCrory DC: Performance characteristics of different modalities for diagnosis of suspected lung cancer: Summary of published evidence. Chest 2003, 1 23: II5S-128S.

4. Charig MJ, Phillips AJ: CT-guided cutting needle biopsy of lung lesions - Safety and efficacy of an out-patient service. Clinical Radiology 2000, 55:964-969.

5. Gasparini S, Ferretti M, Secchi EB, Baldelli S, Zuccatosta L, Gusella P: Integration of transbronchial and percutaneous approach in the diagnosis of peripheral pulmonary nodules or masses. Experience with I,027 consecutive cases. Chest 1995, 108:131-137.

6. Hattori S, Matsuda M, NISHIHAR H, Horai T: Early diagnosis of small peripheral lung cancer - Cytologic diagnosis of very fresh cancer cells obtained by TV-brushing technique. Acta Cytologica 1971, 15:460-467.

7. Lam WK, So SY, Hsu C: Fiberoptic bronchoscopy in the diagnosis of bronchial cancer: Comparison of washings, brushings and biopsies in central and peripheral tumors. Clinical Oncology 1983, 9:35-42.

8. Torrington KG, Kern JD: The utility of fiberoptic bronchoscopy in the evaluation of the solitary pulmonary nodule. Chest 1993, 104:1021-1024.

9. Wallace JM, Deutsch AL: Flexible fiberoptic bronchoscopy and percutaneous needle lung aspiration for evaluating the solitary pulmonary nodule. Chest 1982, 81:665-671.

10. Baaklini WA, Reinoso MA, Gorin AB, Sharafkanch A, Manian P: Diagnostic yield of fiberoptic bronchoscopy in evaluating solitary pulmonary nodules. Chest 2000, I I 7: 1049-1054.

II. Chechani V: Bronchoscopic diagnosis of solitary pulmonary nodules and lung masses in the absence of endobronchial abnormality. Chest 1996, 109:620-625.

12. Estarriol MH, Goday MR, Sanchez MV, Padro XB, Sot MTC, Quetglas FS: Bronchoscopic lung biopsy with fluoroscopy to study 164 localized pulmonary lesions. Archivos de Bronconeumologia 2004, 40:483-488.

13. Naidich DP, Sussman R, Kutcher WL, Aranda CP, Garay SM, Ettenger NA: Solitary pulmonary nodules. CT -bronchoscopic correlation. Chest 1988, 93:595-598.

14. Radke JR, Conway WA, Eyler WR, Kvale PA: Diagnostic accuracy in peripheral lung lesions. Factors predicting success with flexible bronchoscopy. Chest 1979, 76:176-179.

15. Reichenberger F, Weber J, Tamm M, Bolliger CT, Dalquen P, Perruchoud AP, Soler M: The value of transbronchial needle aspiration in the diagnosis of peripheral pulmonary lesions. Chest 1999, I 1 6:704-708.

16. Stringfield JT, Markowitz DJ, Bentz RR, Welch MH, Weg JG: Effect of tumor size and location on diagnosis by fiberoptic bronchoscopy. Chest 1977, 72:474-476.

17. Aristizabal JF, Young KR, Nath H: Can chest CT decrease the use of preoperative bronchoscopy in the evaluation of suspected bronchogenic carcinoma? Chest 1998, I I 3: | 244- 1249.

18. Castella J, Buj J, Puzo C, Anton PA, Burgues C: Diagnosis and staging of bronchogenic carcinoma by transtracheal and transbronchial needle aspiration. Annals of Oncology 1995, 6(Suppl 3):s2I-s24.

19. Pilotti S, Rilke F, Gribaudi G, Spinelli P: Cytologic diagnosis of pulmonary carcinoma on bronchoscopic brushing material. Acta Cytologica 1982, 26:655-660.

20. Bilaceroglu S, Kumcuoglu Z, Alper H, Osma E, Cagirici U, Gunel O, Bayol U, Celikten E, Perim K, Kose T: CT bronchus sign-guided bronchoscopic multiple diagnostic: Procedures in carcinomatous solitary pulmonary nodules and masses. Respiration 1998, 65:49-55

21. Kvale PA, Bode FR, Kini S: Diagnostic accuracy in lung cancer; Comparison of techniques used in association with flexible fiberoptic bronchoscopy. Chest 1976, 69:752-757.

22. Debeljak A, Mermolja M, Sorli J, Zupancic M, Zorman M, Remskar J: Bronchoalveolar lavage in the diagnosis of peripheral primary and secondary malignant lung tumors. Respiration 1994, 61:226-230.

23. de Gracia J, Bravo C, Miravitlles M, Tallada N, Orriols R, Bellmunt J, Vendrell M, Morell F: Diagnostic value of bronchoalveolar lavage in peripheral lung cancer. American Review of Respiratory Disease 1993, 147:649-652. 
24. Gay PC, Brutinel WM: Transbronchial needle aspiration in the practice of bronchoscopy. Mayo Clinic Proceedings 1989, 64:158-162.

25. Mak VHF, Johnston IDA, Hetzel MR, Grubb C: Value of washings and brushings at fiberoptic bronchoscopy in the diagnosis of lung cancer. Thorax 1990, 45:373-376.

26. Mori K, Yanase N, Kaneko M, Ono R, Ikeda S: Diagnosis of peripheral lung cancer in cases of tumors $2 \mathrm{~cm}$ or less in size. Chest 1989, 95:304-308.

27. Pirozynski M: Bronchoalveolar lavage in the diagnosis of peripheral, primary lung cancer. Chest 1992, 102:372-374.

28. Popp W, Rauscher H, Ritschka L, Redtenbacher S, Zwick H, Dutz W: Diagnostic sensitivity of different techniques in the diagnosis of lung tumors with flexible fiberoptic bronchoscope. Comparison of brush biopsy, imprint cytology of forceps biopsy, and histology of forceps biopsy. Cancer 1991, 67:72-75.

29. Zavala DC: Diagnostic fiberoptic bronchoscopy: Techniques and results of biopsy in 600 patients. Chest 1975, 68:12-19.

30. Mclean AN, Semple PD, Franklin DH, Petrie G, Millar EA, Douglas JG: The Scottish multi-centre prospective study of bronchoscopy for bronchial carcinoma and suggested audit standards. Respiratory Medicine 1998, 92: I I I0-II I5.

31. Kacar N, Tuksavul F, Edipoglu O, Ermete S, Guclu SZ: Effectiveness of transbronchial needle aspiration in the diagnosis of exophytic endobronchial lesions and submucosal/peribronchial diseases of the lung. Lung Cancer 2005, 50:22I-226.

32. Govert JA, Kopita JM, Matchar D, Kussin PS, Samuelson WM: Costeffectiveness of collecting routine cytologic specimens during fiberoptic bronchoscopy for endoscopically visible lung tumor. Chest 1996, 109:45I-456.

33. van der Drift MA, van der Wilt GJ, Thunnissen FBJM, Janssen JP: A prospective study of the timing and cost-effectiveness of bronchial washing during bronchoscopy for pulmonary malignant tumors. Chest 2005, I 28:394-400.

34. Karahalli E, Yilmaz A, Turker H, Ozvaran $\mathrm{K}$ : Usefulness of various diagnostic techniques during fiberoptic bronchoscopy for endoscopically visible lung cancer: Should cytologic examinations be performed routinely? Respiration 200I, 68:6II-6I4.

\section{Pre-publication history}

The pre-publication history for this paper can be accessed here:

http://www.biomedcentral.com/1471-2466/8/2/prepub http.//www.biomedcentral.com/1471-2466/8/2 